\title{
LA Chimera di Dino Campana e Altre Chimere
}

Lucia Wataghin

ABSTRACT Il presente articolo contiene considerazioni su esiti e problematiche del riuso dei materiali della tradizione letteraria in una delle più celebrate poesie di Dino Campana, La Chimera.

PAROLE CHIAVE Dino Campana; La Chimera; D'Annunzio.

TESUMO Opresente artigo contém considerações sobre resultados e problemáticas da reutilização de materiais da tradição literária em um dos mais celebrados poemas de Dino Campana, La Chimera.

PAROLE CHIAVE Dino Campana; La Chimera; D’Annunzio.

ABSTRACT The current article brings considerations on the results and problems of reusing material from the literary tradition in one of the most celebrated of Dino Campana's poems, La Chimera.

KLYWORDS Dino Campana; La Chimera; D'Annunzio. 


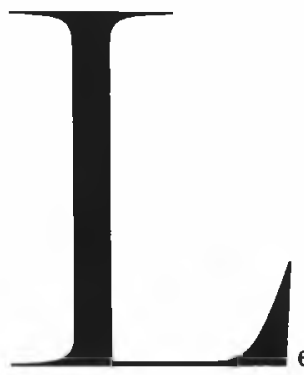

e tracce dell'eredità tardo-ottocentesca e i relativi toni vitalistici e altisonanti sono molto probabilmente il punto più debole, l'aspetto meno tollerato - meno felice - della poesia di Dino Campana. A questoproposito le voci avverse sono numerose: da Fortini, checritica "l'eccesso di sonorità e di fasto" (quando Campana "si affida a cadenze tradizionali", anche in liriche molto celebrate), a Mengaldo, che denuncia "il carattere tardo-ottocentesco e attardato (oltre che caotico) della sua formazione culturale", a Coletti, che mette in guardia contro l'insinuazione, "dentro modalità discorsive e temi della modernità", di "rigurgiti di passato (letterario), con esiti a volte anche ideologicamente inquietanti", a Testa, che ancora più radicalmente, e non solo rispetto a tale eredità, afferma il "congedo pressoché definitivo tra l'autore degli Orfici e gran parte della migliore poesia tardonovecentesca con il suo sistema di lingua, di pensiero e d'attese"1.

Anche i critici meno favorevoli a Campana ritengono che il suo linguaggio attardato sia riscattato dalla modernità in area sintattica - ossia dalla celebratissima qualità della sintassi "stravolta, spesso agrammaticale", che disordina e distorce le comuni sequenze testuali; dal "programmatico disordinamento" su cui insistono Asor Rosa e Coletti ${ }^{2}$, ma resta il fatto che il rapporto con la tradizione orfica e il linguaggio arcaico e intossicante respingono il lettore poco disposto a "pagare il prezzo dell'esoterismo" ${ }^{3}$ per leggere la sua poesia. Eppure buona parte della poesia di Campana, soprattutto nei Canti Orfici, nasce proprio dalla riutilizzazione - spesso in senso espressionistico, stravolgente, deformante - di specifici materiali della tradizione italiana tardo-ottocentesca.

Prendo come esempio una delle liriche più famose e più apprezzate di Campana, La Chimera, in cui si può osservare come una densissima concentrazione di materiali letterari - soprattutto

1. F. Fortini, "La poesia di Campana e il suo mito", in I poeti del Novecento, Bari, Laterza, 1977, pp. 22-30; P. V. Mengaldo, "Dino Campana", in Poeti italiani del Novecento, Milano, Oscar Mondadori, 1978-1990, pp. 275-279; V. Coletti, "Postille a un vecchio saggio su Campana", in Opoesia tu più non tornerai, a cura di M. Verdenelli, Macerata, Quodlibet, 2003, pp. 183-192; E. Testa, “Campana inattuale”, ibidem, pp. 351-355.

2. F. Bandini, "Note sulla lingua poetica di Dino Campana", in Dino Campana alla fine del secolo, a cura di A R. Gentilini, Bologna, Il Mulino, 1999, pp. 39-62 (42); V. Coletti, "Dalla lingua al testo: note linguistiche sui Canti Orfici", ibidem, pp. 63-79 (77). Sul riscatto della "vieillerie poétique" (Rimbaud) nella "alchimia" verbale di Campana vedi Ramat: "Quanti e quali ricorsi "estetici", in Campana! Che abbondanza di "vecchiume" riattato e rilanciato in contesti difformi!", in "Qualche nota per La Chimera", ibidem, pp. 21-38 (31); e A. Corsaro: "echi dannunziani subiscono in Campana un costante rimaneggiamento della cifra stilistica e, pur rinviando a immagini letterariamente caratterizzate, finiscono per produrre una sintassi radicalmente nuova", apud M.A. Grignani, "Momenti della ricezione di Campana", ibidem, pp. 169-187 (181).

3. La formula dí Adorno ("cercare di toccare l'integrità linguistica senza dover pagare il prezzo dell'esoterismo") è usata da Mengaldo per definire il prezzo pagato "dall'ermetismo e anche dai suoi antecedenti prossimi (Onofri, Ungaretti, lo stesso Campana)". P.V. Mengaldo, La tradizione del Novecento, seconda serie, Torino, Einaudi, 2003, p. 15. 
dannunziani - si trasformi in materia del tutto nuova e diversa. La Chimera, una delle prime poesie pubblicate da Campana, esce con il titolo Montagna - La Chimera sul fogliogoliardico "Il Papiro" di Bologna, nel 1912. La prima parte del titolo (Montagna) è eliminata e i versi finali sono radicalmente modificati già nel manoscritto de Il più lungo giorno e nelle edizioni successive dei Canti Orfici, dove compare come primo dei sette notturni che seguono la sezione di apertura, dal titolo La Notte. Nel 1914, anno della prima pubblicazione degli Orfici, in una lettera a Prezzolini (6 gennaio 1914), Campana la presentava così: "Scelgo per inviarle la più vecchia la più ingenua delle mie poesie, vecchia di immagini, ancora involuta di forme: ma Lei sentirà l'anima che si libera"4.

La prima, forte dichiarazione di appartenenza ad una specifica tradizione è enunciata nel titolo, che rimanda a una lunga serie di chimere: da Nerval a Baudelaire a d'Annunzio (a sua volta con molteplici richiami alla tradizione simbolista e decadentista). Nel Novecento, lo stesso tema, in particolare nella sua incarnazione campaniana, sarà ripreso dagli ermetici (in particolare Luzi) e Amelia Rosselli.

Intorno a chimere Nerval e Campana ${ }^{5}$ si rincontrano in ambiente fiorentino: non a caso Les Chimères di Nerval (6 liriche databili negli anni 1852-53), sono ripubblicate in ambito ermetico fiorentino nella traduzione di Alessandro Parronchi (1946); è noto che La Chimera è anche il titolo della rivista ("mensile di letteratura e arte", Vallecchi 1954) a cui collaborarono Luzi, Betocchi, Macrì, Bigongiari, Parronchi; ed è particolarmente al Campana della Chimera che si rifanno Luzi, Parronchi e Bigongiari.Citazioni e riferimenti a Baudelaire ${ }^{6}$ sono numerosi nei Canti Orfici: curiosamente è dal poeta francese che Campana mutua la sua notte michelangiolesca ("toi, grande Nuit, fille de Michel-Ange"); ${ }^{7}$ sulla contiguità e contrapposizione di temi, toni e problemi Baudelaire/Campana dice molto la chimera di Baudelaire (Chacun sa chimère), ${ }^{8}$ una "monstrueuse bête" che impone agli uomini "un invincible besoin de marcher": due chimere, quelle di Baudelaire e di Campana, che sovrapposte ricompongono l'associazione poesia/ pulsione al vagabondaggio che occupa tanta parte della vita di Campana.

Le attestazioni più puntuali di parentela fra le tante chimere di questa tradizione e la chimera di Campana si troveranno comunque piuttosto in ambito italiano che francese. Gli studi campaniani dimostrano con sempre maggiore precisione di dettagli che, benché Campana leggesse anche in lingua originale alcune opere della letteratura europea, il sistema culturale su cui si fonda la sua opera poetica è filtrato in larga misura dall'odiosamato ambiente coevo fiorentino e da d'Annunzio - il suo culto di Wagner e Nietzsche, per esempio, è ispirato da d'Annunzio; ${ }^{9}$ anche il

4. Le mie lettere sono fatte per essere bruciate, a cura di G. Cacho Millet, Milano, All'Insegna del Pesce d'Oro, 1978, pp. $165-166$.

5. Il rapporto di Campana con Nerval è segnato da numerose coincidenze: Nerval, come Campana, narra le notti (Les nuits d'octobre, 1852); la sezione XXIV delle Nuits d'octobre è intitolata "En prison" (e uno dei testi degli Orfici si intitola appunto "Sogno di prigione"); adotta il prosimetro (Petits chateaux de Bohême), come farà Campana; è autore di Promenades e souvenirs (Campana scrive "La petite promenade du poète" e una "Passeggiata in tram in America e ritorno" e si potrebbe dire, come è stato detto di Nerval, che la sua opera fu "une vaste promenade" [H. Lemaitre]) e misticismo, ermetismo, i miti dell'eterno ritorno, del tempo circolare, di Faust, di Orfeo abitano l'immaginazione di entrambi. Le coincidenze biografiche sono inquietanti: viaggiatori infaticabili, affetti da una sorta di mania di vagabondaggio (cosi la definisce Campana), muoiono entrambi all'età di 47 anni dopo aver subito lunghi ricoveri in ospedali psichiatrici.

6. Rilevati nei commenti delle edizioni recenti dei Canti Orfici. Vedi soprattutto Canti Orfici, a cura di Fiorenza Ceragioli, Milano, BUR, 19852004; Canti Orfici eltre poesie, a cura di Neuro Bonifazi, Milano, Garzanti, 1989-1995; Canti Orficie altre poesie, a cura di Renato Martinoni, Torino, Einaudi, 2003. Vedi anche A. Asor Rosa, "Canti Orfici di Dino Campana", in Genus italicum, Saggi sulla identità letteraria italiana nel corso del tempo, Torino, Einaudi, 1997, pp. 683-751 (728-731).

7. C. Baudelaire, L'ideal, apud R. Martinoni, "Introduzione" ai Canti Orficie altre poesie, op. cit., p. XXI. Cf. La Notte di Campana [9].

8. C. Baudelaire, Petits poèmes em prose, ediz. it. Piccoli poemi in prosa, trad. N. Muschitello, Milano, BUR, 1990.

9. G. Bárberi-Squarotti, "Le due chimere: d'Annunzioe Campana", in Dino Campana "una poesia europea musicale colorita", a cura di M. Verdenelli, Macerata, eum, 2007, pp. 17-29 (27-29). Vedi ancheS. Giovannuzzi, "Tra Nietzsche, d'Annunzioe Soffici: la genesi lacerbiana de $I$ più lungo giomo", ibidem, pp. 49-66. Forse l' "Orbo Veggente" d'Annunzio a sua volta si sarà ricordato di Campana autore del notturno $l$ canto della tenebra, scrivendo nel 1916 il Notturno, o Commentario delle Tenebre, in prosa lirica, cronaca di un periodo in cui d'Annunzio rimase temporaneamente cieco. 
mito decadente di Leonardo è dannunziano, condiviso da Baudelaire, Keats e Shelley, Péladan, Walter Pater, Merezkovskij, Angelo Conti, e certamente riproposto all'attenzione di Campana anche dalla rivista di Papini e Prezzolini dal titolo "Il Leonardo" (1903-1907) ${ }^{10}$.

C’è un rapporto fra la Chimera di Campana e la Gorgon ${ }^{11}$ dannunziana (non una chimera, ma una medusa), dal volto pallido e il sorriso leonardesco: ma il pallore della Gorgon è "cupo" e il sorriso è "fulgidissimo e crudele" e in contrasto con la dolcezza dello sguardo, diverso dal semplice "sorriso/di lontananze ignote" della chimera campaniana. Accanto alla Gorgon di d'Annunzio, la Chimera, con la sua spirale di iterazioni parossistiche concentrate sul pallore ("pallido viso"; "eburnea fronte"; "fanciulla esangue"; "mitici pallori"; "la fiamma pallida dei capelli”; "segno del suo pallore") può sembrare una curiosissima serie di variazioni sul tema dell'enfatico incipit di d'Annunzio: "Ella avea diffuso in volto/ quel pallor cupo che adoro" (cf. con l'incipit, dubitativo, di Campana "Non so se tra roccie il tuo pallido/viso m'apparve [...]/").

A prima vista, gli attributi della Chimera di Campana sembrano poco attinenti a qualunque chimera mitologica; tuttavia una suggestiva ipotesi di Silvio Ramat ${ }^{12}$ propone un collegamento fra la Chimera triforme (leone, capra, serpente), tre arti (Pittura, vv. l e 5-6; Danza, vv. 7-9 e Musica, vv. 10, 12 e 15) e tre vergini misteriose (la vergine delle rocce di Leonardo, Proserpina e Santa Cecilia di Raffaello). L'ipotesi di Ramat presuppone il principio della continuità e concordia fra natura e arte postulata da Leonardo; e sono indubitabili, nella Chimera, almeno due riferimenti a Leonardo (la Vergine delle Rocce, sorella della Gioconda).

Nei meandri delle interpretazioni correnti della Chimera la "Regina adolescente" del v. 9 è da rimandare, secondo molti, alla "matrona suadente" alle "barbare travolte regine antiche" dell'Inferno dantesco della Notte [9], e cioè a emblemi della sessualità. La "Regina adolescente" sarebbe dunque una nuova incarnazione di due personaggi della Notte, qui riuniti: la matrona e l'ancella, la Ruffiana e la giovane prostituta ${ }^{13}$. Si potrebbe obiettare che la lirica La Chimera ("Il Papiro" di Bologna, 8 dicembre 1912) e il poema in prosa La Notte (le prime stesure delle sezioni 1-8 escono ne $\mathrm{Il} \mathrm{Goliardo}$ di Genova nel febbraio 1913$)^{14}$ sono concepite e pubblicate separatamente e solo posteriormente riunite nel Più lungo giorno (nell'inverno 1913), il che non esclude comunque una possibile associazione, anche se non di diretta derivazione della prima dalla seconda. Ma la prima ragione (se non altro in ordine cronologico) del misterioso attributo della Chimera ("regina") è da ricondurre piuttosto ai sei versi finali della prima versione della Chimera:

Oggi una fiamma pallida

Entro i capelli viventi

Sul suo profondo pallore

10. Sul mito di Leonardo, nella cultura europea a partire dagli anni ' 87 e in particolare in d'Annunzio, vedi Note a G. d'Annunzio, Versi d'amore e di gloria, Milano, Meridiani Mondadori, 1982-2006, pp. 1136-1137. Anche i temi "notturni" - cari anche a Campana - erano molto attuali nell'ambiente (ideologicamente "inquietante") del "Leonardo". Registro qui una frase di Prezzolini, nella rivista (1904), in tono declamatorio "Verso la notte, verso il mistero, verso il segreto è la nostra azione - tu lo sapevi Novalis che inneggiavi alle Notti" (apud C. Galimberti, Dino Campana, Milano, Mursia, 1967, p. 58).

11. Gorgon, in La Chimera, di G. d'Annunzio. Da questa lirica Campana riprende anche un'associazione fra intercolonni e immagini riflesse nell'acqua, con bellissimo esito (Firenze [Uffizii]). Da Romanza (anche questa nel libro La Chimera di d'Annunzio) l'immagine "Il porto ampio s'addorme,/ stanco d'uman lavoro:", sdoppiata nello stupendo verso della Chimera ("E l'ombre del lavoro umano curve là sui poggi algenti") $e$ in un'altra celebrata immagine ("Il vasto porto si addorme") in Genova.

12. S. Ramat, "Qualche nota per La Chimera", op. cit., pp. 30-31. La prima identificazione della "regina delle primavere spente" con CerereProserpina e della "musica fanciulla esangue" con la Santa Cecilia del dipinto di Raffaello (nella Pinacoteca di Bologna) è di Neuro Bonifazi, Note a Canti Orficie altre poesie, op. cit., p. 135.

13. G. Bárberi Squarotti,"Le due chimere: d'Annunzio e Campana", op. cit., p. 21.

14. F. Ceragioli, "Note" ai Canti Orfici, op. cit., p. 237. 
O Estate che ardi nei cieli

Tu accendi pel suo corpo eburneo:

A la regina dei sogni che appare nei suoi vaghi veli.

Con l'eliminazione di questi versi si è perso un anello della catena di associazioni che formano il personaggio della chimera campaniana: l'anello "regina dei sogni”, che condensa elementi di una precedente chimera dannunziana, anch'essa fortemente avvolta in atmosfera leonardesca: "Io son la Sfinge e sono la Chimera./O tu che sogni, qui ne le mie dita/la trama del tuo sogno è prigioniera"15

Nella versione finale si perde anche in gran parte la viva connotazione sessuale originaria della Chimera; la dannunziana fusione fuoco/pallore, crudeltà e voluttà, è indebolita e condensata nei versi "[Ma per il tuo ignoto poema]/ di voluttà e di dolore". Nel rapporto con d'Annunzio è confermata anche l'origine di un altro degli attributi della Chimera di Campana, la "fiamma pallida" dei capelli, che deriva dalla "fiamma della gran criniera" della Chimera del primo sonetto della Chimera di d'Annunzio ${ }^{16}$. La prima ispirazione della Chimera di Campana è quindi chiaramente legata alla chimera mitologica e a chimere dannunziane.

La Chimera è scritta in codice volutamente ermetico, quasi impenetrabile, e il lettore si dovrà servire di una sorta di campo di significati possibili creato dalle molteplici e non sempre sovrapponibili interpretazioni di critici e commentatori. Un altro esempio di oscurità irrisolta (ancora intorno al termine "Regina"): come intendere i versi "O delle primavere/Spente, per i tuoi mitici pallori/o Regina o Regina adolescente"? Sarà un'allusione a Proserpina, regina degli Inferi "dove fu rapita da Plutone, sparita con lei la primavera" (Bonifazi) e quindi anche alla luna, alla notte, al "poeta notturno" - oppure un'allusione alla "regina... del ricordo" della Notte, in quanto "regina delle primavere trascorse" (Ceragioli)? E ancora: cosa può significare il verso "Io per il tuo divenir taciturno"? È il "futuro silenzioso" (Grillo), è il divenir taciturno di Cecilia, perché "la tradizione del suo martirio la vuole muta" (Ramat), oppure, come scrive Ceragioli, "il poeta è silenzioso, ed è silenzioso anche il farsi della poesia"? E come interpretare i versi 10-15? Raffronti con testi di d'Annunzio offrono sempre nuove chiavi d'accesso al codice: dietro al segreto nascosto nelle labbra della Chimera/Monnalisa si potrebbe leggere anche il segreto della sera di d'Annunzio: il "cerchio delle labbra sinuose" che racchiude l'“ignoto poema/ di voluttà e di dolore", "segnato di linea di sangue", sarebbe una reminiscenza delle colline della Sera fiesolana ("e ti dirò per qual segreto/le colline su i limpidi orizzonti/ s'incùrvino come labbra che un divieto/chiuda [...]") ${ }^{17}$; oltre che un'allusione, naturalmente, al misterioso sorriso delle figure femminili leonardesche. A conferma della vocazione della Chimera al palinsesto - Ramat arriva a parlare di "centonismo"18 -, si osservi anche la corrispondenza di versi memorabili della Chimera con una versione della Cocotte ${ }^{19}$ di Guido Gozzano. Il testo, contenuto in una lettera ad Amalia Guglielminetti, è datato dicembre 1907. Ecco un brano della breve presentazione di Gozzano:

15. D'Annunzio, Al poeta Andrea Sperelli, in La Chimera. Meno tirannica della Sfinge-Chimera dannunziana, da cui forse deriva, appare la notte, "principessa dei sogni segreti", nel terzo notturno dei Canti Orfici, La speranza (sul torrente notturno).

16. G. Bárberi Squarotti, op. cit., p. 22-23.

17. Ibidem.

18. S. Ramat, op. cit., p. 28.

19. G. Gozzano, Il richiamo, in Tutte le poesie, “Nota critica ai testi”, Milano, Meridiani Mondadori, 1980-2006, pp. 708-710. 
"[...] Ho abbozzato una poesia, in endecasillabi e sestine; la poesia è bella, i versi sono brutti. É un richiamo d'una cocotte che conobbi a Cornigliano Ligure, quasi vent'anni fa (del 1889: avevo cinque anni!). Era nostra vicina di casa, perché affittava pei bagni la metà della villa che si affittava noi”

Tono e temi di Gozzano sono lontanissimi dal clima della Chimera, ma Campana riprende quei "brutti" versi (scartati dalla versione definitiva della Cocotte, forse per l'ovvietà del tono declamatorio), cancellandone gli accenti ironici e riportandoli in ambiente sublime: "per i tuoi mitici pallori [...]/Ma per ... [...] Ma per ... [...] Io per .../ Io per .../ Non so se la fiamma pallida/ Fu dei capelli il vivente/ segno del suo pallore,/[...]/E ancora ti chiamo ti chiamo Chimera" Ecco i versi di Gozzano:
"Per quel pallore stanco oggi ti chiamo, per quel pallore stanco io t'amo, t'amo. Ti raggiunga il mio canto di richiamo e ti conduca! Sono qui... T'aspetto!"20 (corsivi miei).

Il rapporto dei testi di Campana con i materiali provenienti dalla tradizione (e in particolare con l'onnipresente d'Annunzio) è spesso cosi ellittico che è difficile e azzardato tentarne la ricostruzione, ma alla luce degli elementi - seppure incerti - cosi raccolti, la figura della Chimera si profila, nella sua programmatica oscurità da canto iniziatico, in una densa rete di associazioni possibili. Come in un dipinto di Leonardo, in primo piano si offre la visione di una figura femminile sfumata e evanescente (e pallida), e sullo sfondo il paesaggio degli ultimi versi. Associazioni con figure meno esangui (matrone, ancelle e regine barbare della Notte) e alcuni attributi fisici (forse il "cerchio delle labbra sinuose", che racchiude l'“ignoto poema/ di voluttà e di dolore") costituiscono la sua dimensione sensuale e sessuale.

La Chimera sarà quindi "una figura femminile ideale" e allo stesso tempo "una figurazione della poesia" (Ceragioli), un' "Illusione eterna dell'uomo, antica e misteriosa ansia d'amore" (Bonifazi), "pura espressione di desiderio" (legata al fuoco della passione poetica), ma anche "una figura femminile di casa di tolleranza", come scrive Asor Rosa, ${ }^{21}$ sarà la luna-Proserpina e "regina del ricordo", sorella della Gioconda, della Vergine delle Rocce, di Santa Cecilia, e "regina dei sogni", o fonte dell'ispirazione, a cui il poeta si rivolge per chiedere accesso ai materiali dell'inconscio.

Sono tutti chiarimenti o ipotesi utili: i riferimenti letterari $o$ alle arti figurative (tranne forse l'ipotetico auto-riferimento, all'ancella/matrona della Notte) si rivelano coerentemente concordi nell'approfondire il clima di rarefazione e lontananza in cui si disegna la figura della Chimera, accrescendo l'effetto ipnotico dell'arabesco. Questo effetto è uno dei risultati più notevoli

20. Un vero e proprio (fulminante) ribaltamento del richiamo di Gozzano alla sua "cattiva Signorina" si trova nei versi di Caproni (che ammette piuttosto di avere alluso alla Traviata: "Parigi, o cara, noi lasceremo,/la vita uniti trascorreremo"): "Su un'eco (stravolta) della Traviata. [per una R.] Dammi la mano. Vieni./Guida la tua guida. Tremo./ Non tremare. Insieme,/ presto Ritorneremo/nel nostro nulla - nel nulla/ (insieme) Rimoriremo." I versi di Gozzano; “Vieni! Sarà come se a te, per mano,/ io riportassi la bella signora,/ la giovinezza, te stessa d'allora!/ Risorgeremo dal tempo lontano!" (corsivi miei).

21. F. Ceragioli, in Canti Orfici, op. cit., p. 281 ; N. Bonifazi, in Canti Orfici ealtre poesie, op. cit., p. 135. Asor Rosa, in "Canti Orfici di Dino Campana", op. cit., p. 718 e in "Dibattito", in Dino campana alla fine del secolo, op. cit., p. 202. Da parte sua Campana, interrogato su questo punto dallo psichiatra Pariani, rispondeva "è una fantasia che avevo, una fantasia qualunque". C. Pariani, Vita non romanzata di Dino Campana, a cura di C. Ortesta, Milano, SE SRL, 2002, p. 42. 
e apprezzati della poesia di Campana: lo stravolgimento o la sconnessione della sequenza logica del periodo, che produce una vertiginosa moltiplicazione di richiami e ambiguità e torsioni sintattiche per cui le immagini sembrano disporsi a spirale; il ritmo anaforico, ricco di ripetizioni e echi ${ }^{22}$. Per contrasto, il disegno dell'arabesco è sospeso negli ultimi sei versi, sei linee parallele (per effetto del polisindeto) dall'enunciazione chiarissima:

"Guardo le bianche rocce le mute fonti dei venti

E l'immobilità dei firmamenti

E i gonfii rivi che vanno piangenti

E l'ombre del lavoro umano curve là sui poggi algenti

E ancora per teneri cieli lontane chiare ombre correnti

E ancora ti chiamo ti chiamo ti chiamo Chimera"

Il paesaggio è introdotto gradualmente, come naturale ampliamento del punto di vista del poeta, ma resta sorprendente: non senza ragione questi versi sono definiti da Asor Rosa "sei tra i versi più belli del Novecento italiano"23.

Per finire, un accenno a un altro paesaggio e un Campana antisublime, antidannunziano e forse anche antichimerico, in un frammento in prosa - un altro palinsesto - di cui Bărberi-Squarotti ${ }^{24}$ ha studiato i densi rapporti con testi dannunziani. L'intero frammento è una ulteriore conferma della varietà di reazioni suscitate in Campana dalla complessa relazione con d'Annunzio, che resta per lui un fortissimo punto di riferimento, spesso oggetto di polemici rifiuti, ma spesso citato in modi e con effetti imprevisti. Le due frasi finali del frammento costituiscono uno dei più bei paesaggi di Campana, con annesso autoritratto del poeta autoironico e spassionato che osserva da lontano una Gorgona (isola e Chimera?):

"Appiccicato alla spallina del passeggio guardo il mare senza parole come io sono senza pensiero. La Gorgona è un dosso sul mare abbandonato laggiù nei tramonti.”

22. Sono questi gli aspetti della Chimera di Campana portati all'estremo nella lettura di Amelia Rosselli, che estende le violazioni alle norme dell'italiano (dalla sintassi, violata da Campana, al lessico e alla grammatica); cheesaspera "fino alla resa patologica" la figura della ripetizione (N. Lorenzini, p. 192) e più in generale esprime con forza nella sua Chimera quello che Giudici definisce il "derèglement che è la "sua" lingua italiana impazzita con superiore sapienza", intorno ai temi dell'amore, della malattia, del lutto, della morte: "Non so se tra le pallide brume il tuo sorriso/m'apparve in una bruma tiepida e distesa, $\mathrm{ma}$ / io morii dal male che sorse dalla tua bocca e/dal tuo tiepido sorriso infatuato. Non se se/tra il male che mi vuole e il tuo sorriso esista/la pietra puntata dalla differenza: se gemelle/le nostre anime sono, non so come accordarle/ al tuo suono flebile non vedo la luna apparire/di tra gli spuntati roccioni delle mie abitudini./Non so se tra le pallide rocce il tuo sorriso/ m'apparve, o sorriso di lontananze ignote, o se/ tra le tue pallide gote stornava il ritornello/ che la tempesta ruppe su la testa rotta. Non/ so se tra le pallide rocce m'apparve, un sorriso/di lontananze ignote, non so se di tra le pallide/bocche se di tra le pallide smorfie dei viventi/io rimarrò ancora: non so se tra le pallide gonfie/ tenebre de la miseria tu entrerai a festare [...]". Il testo fa parte del poemetto La Libellula, del 1958. Apud N. Lorenzini, “Conclusioni”, in Dino Campana alla fine del secolo, op. cit., p. 192.

23. A Asor Rosa, "Canti Orfici di Dino Campana”, op. cit., p. 718.

24. Bárberi Squarotti, Giorgio, "Le due Chimere: d'Annunzio e Campana”, op. cit., pp. 17-19. Il frammento citato dal critico è tratto dagli Inediti (Taccuini, abbozzi e carte varie) di Campana. 